\title{
Survival of Colorectal Cancer in the Presence of Competing- Risks - Modeling by Weibull Distribution
}

\author{
Ahmad Reza Baghestani',Tahoura Daneshvar ${ }^{1 *}$, Mohamad Amin Pourhoseingholi², \\ Hamid Asadzadeh ${ }^{2}$
}

\begin{abstract}
Background: Colorectal cancer (CRC) is the commonest malignancy in the lower gastrointestinal tract in both men and women. It is the third leading cause of cancer-dependent death in the world. In Iran the incidence of colorectal cancer has increased during the last 25 years. Materials and Methods: In this article we analyzed the survival of 447 colorectal patients of Taleghani hospital in Tehran using parametric competing-risks models. The cancers of these patients were diagnosed during 1985 - 2012 and followed up to 2013 . The purpose was to assess the association between survival of patients with colorectal cancer in the presence of competing-risks and prognostic factors using parametric models. The analysis was carried out using $R$ software version 3.0.2. Results: The prognostic variables included in the model were age at diagnosis, tumour site, body mass index and sex. The effect of age at diagnosis and body mass index on survival time was statistically significant. The median survival for Iranian patients with colorectal cancer is about 20 years. Conclusions: Survival function based on Weibull model compared with Kaplan-Meier survival function is smooth. Iranian data suggest a younger age distribution compared to Western reports for CRC.
\end{abstract}

Keywords: Survival analysis - colorectal cancer - parametric competing-risks

Asian Pac J Cancer Prev, 17 (3), 1193-1196

\section{Introduction}

Colorectal cancer (CRC) is the commonest malignancy in the gastrointestinal tract (Chan et al., 2010) and common diagnosed cancer in both men and women. It is the third leading cause of cancer dependent death in the world and there are one million new cases diagnosed per year (Ranjbar1 et al., 2014). The highest incidence rates of colorectal cancer are located in Europe, North America, and Oceania. In contrast, the lowest rates are observed in Asia, Africa, and South America (Center et al., 2009). In Eastern Asian countries such as China, Japan, South Korea and Singapore have experienced a two- to fourfold increase in incidence in recent decades (Sung et al., 2005). In recent years, a population based study in United States has shown an increase in the incidence of colorectal cancer in the young (Chan et al., 2010). During 20022006, 10,284 new cases of invasive colorectal cancer were diagnosed in Ireland (McDevitt et al., 2013).

In the recent decade incidence trends of colorectal cancer have differed between populations so that the incidence rate has decreased in USA, slightly increasing or constant in Europe and highly increasing in most Asian countries (Abdifard et al., 2013).

During the last 25 years the incidence of colorectal cancer has increased in Iran (Mousavi et al., 2009). Also it is the fifth cause of cancer in men and the third in women (Pourhoseingholi and Zali, 2012). Iranian data suggest a younger age distribution for CRC compared to Western reports (Azadeh et al., 2008). Studi show that Kermanshah in Western Iran had the highest risk of colorectal cancer incidence (Abdifard et al., 2013).

Survival analysis is the modelling of time to event of death to evaluate the effects of treatment on survival time (David G Kleinbaum, 2012). There are situations in which a studied person can experience one of several different types of events. Occurrence of one event type prevents others from occurring. In this situation the best way to analyse survival time is Competing-risks model (Crowder, 2010). Here survival of a CRC patient means the years between diagnosis of disease and the death due to this cancer.

Although semi parametric method is the most common technique in competing-risks survival analysis, the parametric model also leads to some benefit (Baghestani et al., 2010). Researchers in medical science often tend to prefer semi parametric over parametric models because they require less assumptions (Moeschberger and Klein, 2003). When these parametric models provide a good fit to data, they tend to give more precise estimates of the 
quantities of interest because these estimates are based on fewer parameters (Baghestani et al., 2010).

In general the purpose of this study was to assess the association between survival of patients with colorectal cancer and prognostic factors using parametric competingrisks model and obtaining the survival of colorectal patients

\section{Materials and Methods}

In recent years a pilot screening has been performed on individuals at high risk of familial CRC by the Research Institute for Gastroenterology and Liver Disease, Shahid Beheshti University of Medical Science. Colorectal cancer patients from across the country are invited to the hospital for screening, their family members included in screening program as well and then they are annually followed for survival. In this study 447 patients were selected as a sample of these patients in Iran.

The cancer of these patients was diagnosed during 1985-2012 and followed up to 2013. To obtain information about patients, telephone calls were made. The demographic features which were extracted from hospital documents were included in the analysis as prognostic variables; item, such as age at diagnosis, sex, tumour site and body mass index known as "BMI". The body mass index "BMI" is defined as the individual's body mass divided by the square of their height. $\left(\mathrm{Kg} / \mathrm{m}^{2}\right)$ The World Health Organisation "WHO", uses the BMI to help decide whether people are too fat or too thin. Our aim is to see whether it has statistically significant effect on colorectal patient's survival time or not?

In recent follow ups it is found that about $14 \%$ of colorectal patients died of other causes of death, such as myocardial infarction and stomach cancer so we analyzed the data by using competing-risks models and because of the some benefits of parametric models we have used parametric competing-risks.

Prognostic variables were entered in a parametric model called Weibull distribution. The analysis was carried out using $\mathrm{R}$ software version 3.0.2

\section{Results}

A total number of 447 patients with colorectal cancer were included in the study. The cancer of these patients was diagnosed during 1985 - 2012 and followed up to 2013. After recent follow ups it is confirmed that 160 cases were alive, 113 cases had died due to colorectal cancer, 174 cases were censored. There were $55 \%$ male cases so 1.24:1(male: female) sex ratio were found.

The age ranges were from 18 to 83 years at the time of diagnosis but most of the patients $(51 \%)$ at the time of the diagnosis were between 41 and 60 years old. The mean age at the time of diagnosis is $53 \pm 14$.

Tumour site was classified into two groups "colon" and "rectum". The tumours of 51 percent of these patients were located in rectum.

The mean of these patients's BMI is $25 \pm 4.2$.

The result of using Weibull distribution is shown in table 1. Based on this model the effects of age at diagnosis
$S(t)$

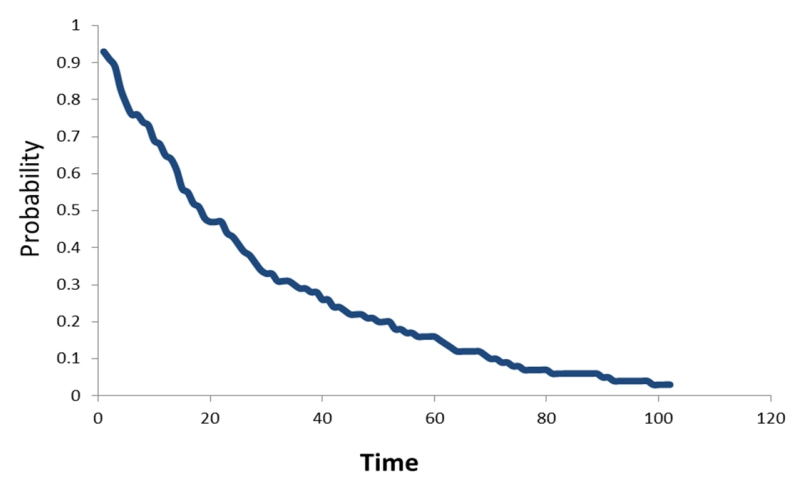

Figure 1. Survival Function of 447 Colorectal Patients Based on Weibull Distribution

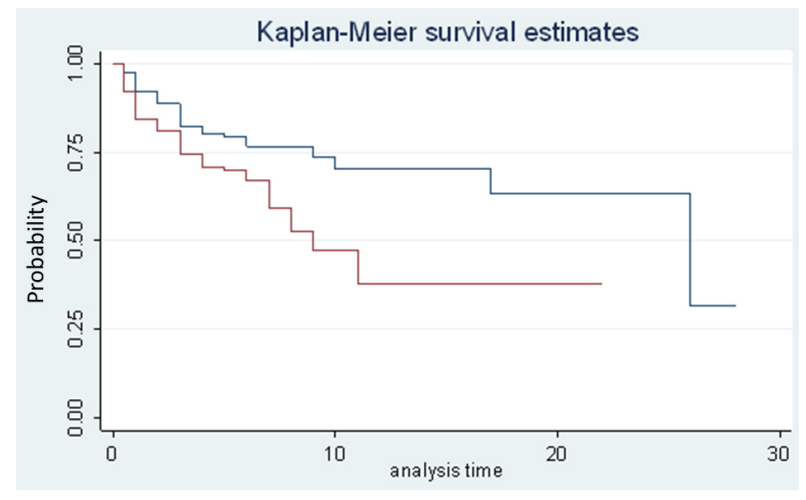

Figure 2. Survival Functions of 447 Colorectal Patients Separated by age at Diagnosis

Table 1. Competing Risk Regression of 446 Cases of Colorectal Cancer

\begin{tabular}{lccc}
\hline Variables & Coefficient & $\mathrm{sd}$ & $\mathrm{P}$ - value \\
\hline BMI & -0.016 & 0.005 & $0.00^{*}$ \\
Age At Diagnosis & 0.019 & 0.002 & $0.00^{*}$ \\
Tumour Site & 0.02 & 0.17 & 0.45 \\
sex & -0.2 & 0.2 & 0.15 \\
\hline
\end{tabular}

and BMI are statistically significant on survival time, but the others do not have any statistically significant effect on the colorectal patient's survival time.

The hazard ratio of age at diagnosis for these patients is 1.02 which means by increasing age at the time of diagnosis risk of patient's death due to colorectal cancer would increase. The hazard ratio of BMI for these patients is 0.98 which means a decrease in BMI would increase the risk of patient's death due to colorectal cancer.

Figure 1 shows the survival of colorectal patients based on Weibull distribution. The median survival time is about 20 years. In figure 2 the survival of colorectal patients is separated by the age at the time of diagnosis. This figure is based on Kaplan-Meier method which is the most common method to analyse survival data. The median survival time for patients whose time of diagnosis is less than 53years old is about 26 years and the median survival time for patients whose time of diagnosis is more than 53years is about 8 years. 


\section{Discussion}

Colorectal cancer is one of the important public health problems and it is expected to become a major health problem globally as a result of high and increasing incidence and rate (Moradi et al., 2009).

The purpose of this study was to assess the association between survival of patients with colorectal cancer and prognostic factors using parametric models and also obtaining the survival of colorectal patients. Based on Weibull model age at diagnosis of the cancer has significant effect on these patients survival time which is in line with the Li's study (Li et al., 2013). Also Morrison's study indicated only age was significant determinant of both colon and rectum cancer (Morrison et al., 2013). One can conclude a patient whose disease has been diagnosed before the age of 53 has a longer life in comparison to one whose disease has been diagnosed after the age of 53 .

Survival curve based on Weibull model (Table1) compared with Kaplan-Meier survival function is smooth (Maller and Zhou, 2002).

The mean age at the time of diagnosis is $53 \pm 14$ which is approximately the same as the results of Golfam's study (Golfam et al., 2013). This study shows that the Iranian data still suggest a younger age distribution compared to Western report for CRC. Appropriate screening strategies especially in relatives of the patient should be considered (Pourhoseingholi and Zali, 2012). This younger age distribution at the time of diagnosis makes different survival compared to other countries for instance Khattak et al found 20.3 months as a median survival of 1207 colorectal patients in south Australlian (Khattak et al., 2012) but here the overall median survival time for these colorectal patients is 20 years but the median survival time for these patients whose time of diagnosis is before the age of 53 is 25 years while the median survival time for these patients whose time of diagnosis is after the age of 53 is about 8 years.

There is evidence to support the screening of average risk individuals over the age of 50 to detect and prevent colorectal cancer. Screening of these people can reduce mortality by identifying cancer at an early stage (Labianca and Merelli, 2010).

The sex ratio of 1.24:1(male: female) is found for colorectal cancer which is approximately equal to the results of Safaee's research (Safaee et al., 2012). IIn this study the effect of sex is not statistically significant on survival time, so sex does not have statistically significant effect on survival time which is in line with the Li's study as well and also is in contrary with Heidarnia's study in which age and gender both have statistically significant effect on colorectal patient's survival time (Heidarnia et al., 2013).

In these data there are five different failures; dying of CRC cancer, dying due to myocardial infarction, dying by stomach cancer or kidney and lung disease. Surly in other researches the death of all patients were not do to colorectal cancer, so we suggest the best way to analyse the patient's survival time is competing-risks method. By using this method our results shows slightly different results compared to previous researches such as Safaee's study (Azadeh et al., 2008).

BMI had statistically significant effect on survival time; decrease in BMI would increase the risk of patient's death due to colorectal cancer. It is in contrary with Nilson's study, in their research they had found no relationship between BMI and risk of colorectal cancer (Nilsen and Vatten, 2001). Caan et al indicated in their research that a slight increase in BMI would increase the risk of colorectal cancer (Caan et al., 1998) so one can conclude a increase in BMI would increase the risk of colorectal cancer in human, but if a colorectal patient lose weight, would die sooner in comparison to one who did not lose weight.

Baghestani et al analysed these data by semi parametric competing-risks method. They have indicated that only age at diagnosis has statistically significant result on survival time (Baghestani et al., 2014).

\section{Acknowledgements}

This study received financial support from Research Centre for Gastroenterology and Liver Diseases, Taleghani Hospital, Shahid Beheshti University of Medical Sciences. This study is derived from the master's thesis

\section{References}

Abdifard E, Ghaderi S, Hosseini S, Heidari M (2013). Incidence trends of colorectal cancer in the West of Iran during 20002005. Asian Pac J Cancer Prev, 14, 1807-11.

Azadeh S, Moghimi-Dehkordi B, Fatem SR, et al (2008). Colorectal cancer in Iran: an epidemiological study. Asian Pac J Cancer Prev, 9, 123-6.

Baghestani AR, Daneshvar T, Pourhoseingholi MA, Asadzade H (2014). Survival of colorectal cancer patients in the presence of competing-risk. Asian Pac J Cancer Prev, 15, 6253.

Baghestani AR, Hajizadeh E, Fatemi SR (2010). Parametric model to analyse the survival of gastric cancer in the presence of interval censoring. Tumori, 96, 433-7.

Caan B, Coates A, Slattery M, et al (1998). Body size and the risk of colon cancer in a large case-control study. Int J Obesity Related Metabolic Disorders, 22, 178-84.

Center MM, Jemal A, Smith RA, Ward E (2009). Worldwide variations in colorectal cancer. CA Cancer J Clin, 59, 366-78.

Chan K, Dassanayake B, Deen R, et al (2010). Young patients with colorectal cancer have poor survival in the first twenty months after operation and predictable survival in the medium and long-term: analysis of survival and prognostic markers. World J Surg Oncol, 8, 20840793.

Crowder MJ. Classical competing risks. CRC Press, 2010.

David G Kleinbaum MK. Survivale analysis. springer. Issue, Page ??

Golfam F, Golfam P, Neghabi Z (2013). Frequency of all types of colorectal tumors in the patients referred to selected hospitals in Tehran. Iranian Red Crescent Med J, 15, 473.

Heidarnia MA, Monfared ED, Akbari ME, et al (2013). Social determinants of health and 5-year survival of colorectal cancer. Asian Pac J Cancer Prev, 14, 5111-6.

Khattak MA, Martin HL, Beeke C, et al (2012). Survival differences in patients with metastatic colorectal cancer and with single site metastatic disease at initial presentation: results from South Australian clinical registry for advanced colorectal cancer. Clin Colorectal Cancer, 11, 247-54.

Labianca R, Merelli B (2010). Screening and diagnosis for 
colorectal cancer: present and future. Tumori, 96, 889 .

Li XP, Xie ZY, Fu YF, et al (2013). Colorectal cancer concealment predicts a poor survival: a retrospective study. Asian Pac J Cancer Prev, 14, 4157-60.

Maller RA, Zhou X (2002). Analysis of parametric models for competing risks. Statistica Sinica, 12, 725-50.

McDevitt J, Sharp L, MacDonald D, Dwane F, Comber H (2013). Factors affecting receipt of a medical card in a cohort of colorectal cancer patients, 2002-2006. Irish Med $J, \mathbf{1 0 6}, 110-3$.

Moeschberger ML, Klein J (2003). Survival analysis: Techniques for censored and truncated data: Statistics for Biology Health. Springer.

Moradi A, Khayamzadeh M, Guya M, Mirzaei HR, Salmanian $\mathrm{R}$, Rakhsha A, et al (2009). Survival of colorectal cancer in Iran. Asian Pac J Cancer Prev, 10583-6.

Morrison DS, Parr CL, Lam TH, et al (2013). Behavioural and metabolic risk factors for mortality from colon and rectum cancer: analysis of data from the Asia-pacific cohort studies collaboration. Asian Pac J Cancer Prev, 14, 1083-7.

Mousavi SM, Gouya MM, Ramazani R, Davanlou M, Hajsadeghi $\mathrm{N}$, Seddighi Z (2009). Cancer incidence and mortality in Iran. Ann Oncol, 20, 556-63.

Nilsen TL, Vatten LJ (2001). Prospective study of colorectal cancer risk and physical activity, diabetes, blood glucose and BMI: exploring the hyperinsulinaemia hypothesis. British J Cancer, 84, 417.

Pourhoseingholi MA, Zali MR (2012). Colorectal cancer screening: Time for action in Iran. World J Gastrointestinal Oncol, 4, 82-3.

Ranjbar1, R, Saberfar E, Shamsaie A, Ghasemian E (2014). The aetiological role of human papillomavirus in colorectal carcinoma: an iranian population-based case control study. Asian Pac J Cancer Prev, 15, 1521-5.

Safaee A, Fatemi SR, Ashtari S, Vahedi M, Moghimi-Dehkordi B, Zali MR (2012). Four years incidence rate of colorectal cancer in Iran: a survey of national cancer registry dataimplications for screening. Asian Pac J Cancer Prev, 13, 2695-8.

Sung JJ, Lau JY, Goh K, Leung W (2005). Increasing incidence of colorectal cancer in Asia: implications for screening. Lancet Oncol, 6, 871-6. 